\title{
To wet or not to wet? Dispersion forces tip the balance for water-ice on metals
}

\author{
Javier Carrasco ${ }^{1,2}$, Biswajit Santra ${ }^{2}$, Jiří Klimeš ${ }^{1}$, Angelos Michaelides ${ }^{1}$ \\ ${ }^{1}$ London Centre for Nanotechnology and Department of Chemistry, \\ University College London, \\ London WC1E 6BT, UK \\ ${ }^{2}$ Fritz-Haber-Institut der Max-Planck-Gesellschaft, \\ Faradayweg 4-6, D-14195 Berlin, Germany
}

\begin{abstract}
Despite widespread discussion, the role of van der Waals dispersion forces in wetting remains unclear. Here we show that non-local correlations contribute substantially to the water-metal bond and that this is an important factor in governing the relative stabilities of wetting layers and 3D bulk ice. Due to the greater polarizability of the substrate metal atoms, non-local correlations between water and the metal exceed those between water molecules within ice. This sheds light on a long-standing problem, wherein common density functional theory exchange-correlation functionals incorrectly predict that none of the low temperature experimentally characterized ice-like wetting layers are thermodynamically stable.

PACS numbers: $\quad$ 68.43.Bc, 68.43.Fg, 82.65.+r,
\end{abstract}


Water covers almost all solid surfaces under ambient conditions. As such, interfacial water is of crucial importance to an endless list of problems in the physical and chemical sciences. Examples include heterogenous ice nucleation on aerosol particles of relevance to the atmospheric sciences, the flow of confined water through membranes and pores in connection with geology and waste water treatment, or the response and reactivity of water to electrochemical fields [1, 2]. A prerequisite to understand these varied phenomena is the seemingly simple task of establishing how the water molecules are arranged at the interface, i.e., what the water overlayer structure is. Characterizing water overlayer structures is, however, a challenging task and despite thousands of publications on the chemical physics of water at interfaces only a handful of determinations have been accomplished to date [1, 2]. These have all been on well-defined atomically smooth substrates - mostly metal surfaces - under ultra-high vacuum (UHV) conditions, to which the whole arsenal of surface science experimental probes can be applied [1, 2]. Instrumental in recent determinations has been density functional theory (DFT), and indeed there is now an almost symbiotic relationship between DFT and scanning tunnelling microscopy (STM), with DFT being used to provide structural models with which the experiments can be interpreted. Notable examples where DFT has been crucial in unravelling water overlayer structures include water in the submonolayer regime on $\operatorname{Pd}(111)[3], \mathrm{Cu}(110)$ [4], and $\operatorname{Pt}(111)$ [5].

Despite the undeniable value of DFT in helping to understand water on metals, there is an important and widely discussed problem [1, 3-21]. Specifically, the structures identified with widely used generalized gradient approximation (GGA) functionals such as PBE or PW91 are consistently found to be less stable than ice Ih (the most common form of ice) [319]. This suggests that the intact water overlayers should simply not form under equilibrium conditions. This is an uneasy position for the role of DFT in water adsorption studies and it casts doubt on the structural characterizations themselves. Since the typical GGAs used in such studies do not account properly for van der Waals (vdW) dispersion forces, it would of course be interesting and important to know what role vdW forces play in water adsorption. Apart from two limited attempts to address this issue [22, 23], and widespread discussion [1, 3-21], no detailed study on the role of dispersion forces in realistic water adlayer structures on metals has been reported. Thanks to the development of xc functionals which explicitly account for dispersion, such as the non-local vdW density functional (vdW-DF) of Dion et al. [24] and its offspring [25], it is now possible to tackle this question head on. 
Here we report a study in which the role of dispersion in water metal bonding is examined in detail. To this end we apply a newly developed non-local functional to two of the most widely studied water-ice adsorption systems, namely water on $\mathrm{Cu}(110)$ and $\mathrm{Ru}(0001)$. We find that non-local correlations contribute substantially to the water-metal bond and that this is an important factor in governing the relative stabilities of wetting layers and 3D bulk ice. The fact that dispersion forces favor 1D and 2D wetting layer structures over 3D bulk ice is simply due to the much larger polarizability of the substrate metal atoms. This study highlights the key role dispersion plays in wetting and how non-local functionals ameliorate the problem common GGAs have with experimentally characterized wetting layers on metal surfaces. Since quantitative first principles predictions of wetting and ice growth on metals are still beyond reach, some of the outstanding problems are also briefly discussed.

DFT calculations were performed with a modified version of the VASP 5.2 code [26, 27], which includes our own self-consistent implementation of the non-local van der Waals density functionals [28]. Results are reported for PBE [29] and a modified version of vdW-DF, referred to as "optB88-vdW" [25]. The difference between the original vdw-DF of Dion et al. [24] and optB88-vdW is merely in the exchange functional, with the optB88 exchange functional yielding more accurate interaction energies than the original choice of revPBE [25]. The $\mathrm{Cu}(110)$ and $\mathrm{Ru}(0001)$ surfaces were modelled with slabs of between 4 and 6 layers thickness separated by $14 \AA$ of vacuum. Bulk ice was modelled using Hamann's twelve water molecule ice cell [30]. Core electrons were treated with the projector augmented wave (PAW) method [31], whilst the valence electrons $\left(3 d^{10} 4 s^{1}\right.$ and $4 d^{7} 5 s^{1}$ for $\mathrm{Cu}$ and $\mathrm{Ru}$, respectively) were expanded in a plane wave basis with a $600 \mathrm{eV}$ cut-off energy. Adsorption energies per water molecule were computed from $E_{\text {ads }}=\left(E^{\text {tot }}\left[\mathrm{H}_{2} \mathrm{O} / \mathrm{M}\right]-E^{\text {tot }}[\mathrm{M}]-n E^{\text {tot }}\left[\mathrm{H}_{2} \mathrm{O}\right]\right) / n$; where $E^{\text {tot }}\left[\mathrm{H}_{2} \mathrm{O} / \mathrm{M}\right]$ is the total energy of the $n$ water molecule adsorption system, $E^{\text {tot }}[\mathrm{M}]$ is the total energy of the relaxed bare metal slab and $E^{\text {tot }}\left[\mathrm{H}_{2} \mathrm{O}\right]$ is the total energy of an isolated gas phase $\mathrm{H}_{2} \mathrm{O}$ molecule. Likewise the lattice energy of bulk ice is obtained by subtracting the total energy of the $n \mathrm{H}_{2} \mathrm{O}$ ice supercell from the total energy of $n$ gas phase $\mathrm{H}_{2} \mathrm{O}$ molecules. Zero point corrected versions of these quantities were also computed from harmonic vibrational frequencies. Some of the energy differences between the structures considered here are very small. However, we have carried out extensive convergence tests (some of which are reported in Tables SI and SII of the supporting information) which give us confidence that our chosen computational set-up is sufficiently close to convergence that 
none of the conclusions reached here would be altered if yet more accurate settings were used.

Let us first consider the wetting of $\mathrm{Cu}(110)$. Below $140 \mathrm{~K}$ and for a range of temperatures and coverages in the submonolayer regime, water forms 1D chains which run perpendicular to the $\mathrm{Cu}(110)$ ridges. These chains have been observed by a number of groups [4, 32, 33] and although caution must in general be exercised in considering how kinetics dictates water film morphology in low temperature UHV experiments, all existing experimental evidence suggests that the $1 \mathrm{D}$ chains are the thermodynamically stable phase for water on $\mathrm{Cu}(110)$ at low coverage. The structure of the chains has recently been characterized and shown to be a periodic arrangement of water pentagons on the basis of STM, infra-red spectroscopy, and DFT [4]. Hence the 1D chains provide an excellent opportunity to understand the relative stability of bulk ice with a well-defined wetting layer and to explore the role of dispersion forces in wetting on metals.

The structure of the water chains is shown in Fig. 1(a). They are comprised of a face sharing arrangement of water pentagons with two types of water molecules: flat-lying molecules bonded directly to $\mathrm{Cu}$ surface atoms along the ridges and upright molecules over the troughs in the (110) surface which interact relatively weakly with the substrate. Fig. 2 summarizes the adsorption energies obtained, along with the corresponding lattice energies of bulk ice. Results with and without zero point energies are reported. The first observation is that PBE predicts bulk ice to be more stable than the pentagon-based wetting layer. Specifically, the energy of the adsorbed structure is $92 \mathrm{meV} / \mathrm{H}_{2} \mathrm{O}$ less stable than the lattice energy of bulk ice. Accounting for the zero point energy difference between the overlayer and ice is not sufficient to correct the ordering of the two states, although it does push things in the right direction by reducing the preference for bulk ice to $63 \mathrm{meV} / \mathrm{H}_{2} \mathrm{O}$. This incorrect preference for ice is precisely the problem that has been observed many times before for pure water overlayers computed with PBE and PW91 [1, 3-21]. Moving to the optB88-vdW functional, we find that it does indeed give the correct energy ordering, predicting that the overlayer is marginally $\left(13 \mathrm{meV} / \mathrm{H}_{2} \mathrm{O}\right)$ more stable than bulk ice at the total energy level and $39 \mathrm{meV} / \mathrm{H}_{2} \mathrm{O}$ more stable when zero point energies are accounted for. Thus a stable wetting layer is found in agreement with the experimental observations. The structure of the overlayers obtained with PBE and optB88-vdW are very similar (all hydrogen bonds between water molecules and water-metal distances are within $0.02 \AA$ ), which indicates 

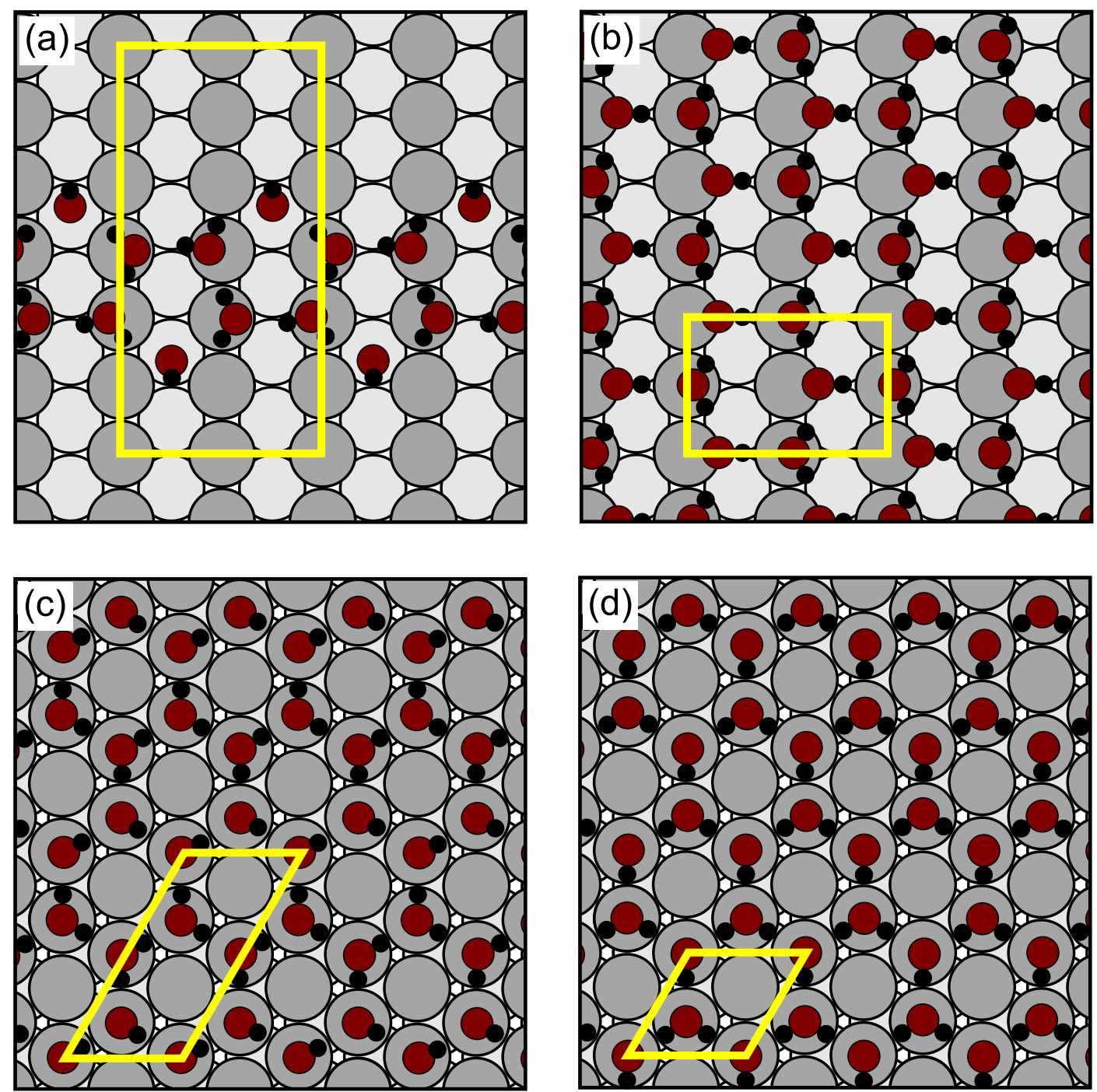

FIG. 1. Top views of water overlayers: (a) a pentagonal chain on $\mathrm{Cu}(110)[4]$; (b) H-down bilayer on $\mathrm{Cu}(110)$; (c) extended chains on $\mathrm{Ru}(0001)$ with flat-lying and H-down molecules [15]; and (d) H-down bilayer on $\mathrm{Ru}(0001)$. Black, red, dark gray, and light gray circles represent $\mathrm{H}, \mathrm{O}$, and $\mathrm{Cu}$ $(\mathrm{Ru})$ in the first and second layer, respectively. For molecules in the H-down configuration the $\mathrm{H}$ atoms beneath the oxygens are not visible. The unit cells used are indicated.

that whilst dispersion is important for the absolute adsorption energy of water it is of less importance to the atomic structure.

As a consistency check, we compared the adsorption energy of the pentagonal overlayer with extended 2D bilayers (both the "H-down", Fig. 1(b), and "H-up" bilayers considered for 


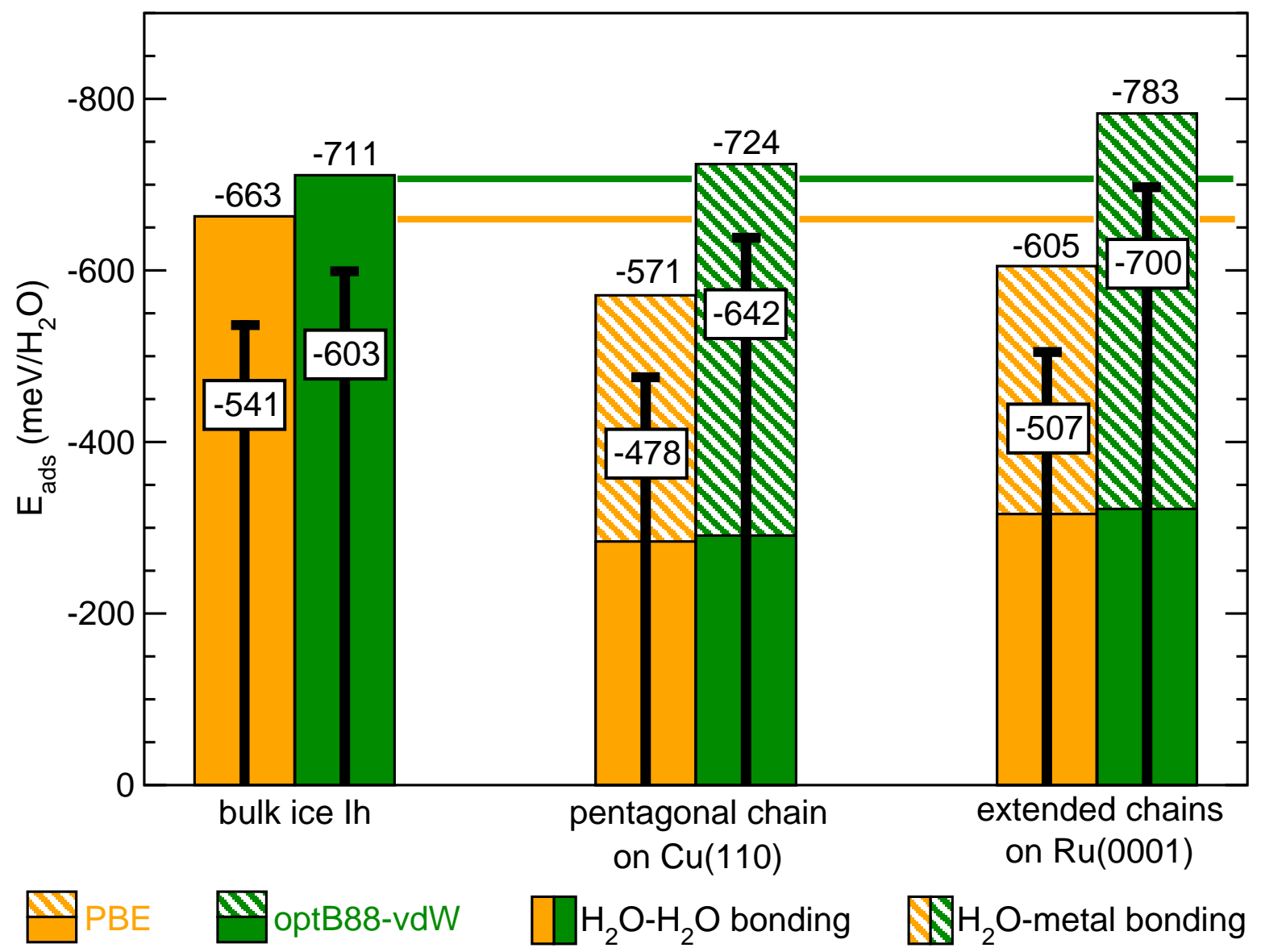

FIG. 2. Adsorption energies of water overlayers on $\mathrm{Cu}(110)$ and $\mathrm{Ru}(0001)$ compared to the lattice energy of bulk ice Ih using PBE and optB88-vdW. The adsorption energy is also decomposed in to water-water and water-metal bonding. The thin centered black bars and associated numbers are the adsorption/lattice energies corrected for zero point energies.

this system before [14]) and alternative 1D chain models [4]. Irrespective of the functional used the pentagonal chain structure remains the most stable overlayer. Therefore, the relative energies of the various overlayers are not strongly affected by dispersion forces, which explains why PBE and PW91 have had success in predicting water structures on metals. Finally, explicit consideration of finite temperature vibrational free energy effects does not alter the conclusions drawn here and when these effects are accounted for the pentagons remain more stable than bulk ice (Fig. SII).

Regarding now the wetting of $\mathrm{Ru}(0001)$, Hodgson and co-workers have shown that between 140 and $160 \mathrm{~K}$ a 2D wetting layer of intact water molecules forms, which upon heating or exposure to electrons transforms in to a mixed $\mathrm{H}_{2} \mathrm{O}-\mathrm{OH}$ overlayer [15, 19]. At 0.67 
TABLE I. Different contributions to the optB88-vdW adsorption energy $\left(E_{\text {ads }}\right)$ of the pentagons on $\mathrm{Cu}(110)$ and the extended chain structure on $\mathrm{Ru}(0001)$ : water-water bonding $\left(E_{\text {gas }}^{\mathrm{H}_{2} \mathrm{O}-\mathrm{H}_{2} \mathrm{O}}\right)$, watermetal bonding $\left(E_{\text {ads }}^{\mathrm{H}_{2} \mathrm{O}-\mathrm{M}}\right)$, non-local correlation contribution to the adsorption energy $\left(E_{\text {ads }}^{\text {nlc }}\right)$, nonlocal correlation contribution to water-water bonding $\left(E_{\text {gas }}^{\mathrm{H}_{2} \mathrm{O}-\mathrm{H}_{2} \mathrm{O} \text {,nlc }}\right)$, and non-local correlation contribution to water-metal bonding $\left(E_{\mathrm{ads}}^{\mathrm{H}_{2} \mathrm{O}-\mathrm{M}, \mathrm{nlc}}\right)$. Zero point energy corrected values are given in parenthesis. All values in $\mathrm{meV} / \mathrm{H}_{2} \mathrm{O}$.

\begin{tabular}{ccccccc}
\hline \hline & $E_{\text {ads }}$ & $E_{\text {gas }}^{\mathrm{H}_{2} \mathrm{O}-\mathrm{H}_{2} \mathrm{O}}$ & $E_{\text {ads }}^{\mathrm{H}_{2} \mathrm{O}-\mathrm{M}}$ & $E_{\text {ads }}^{\text {nlc }}$ & $E_{\text {gas }}^{\mathrm{H}_{2} \mathrm{O}-\mathrm{H}_{2} \mathrm{O}, \text { nlc }}$ & $E_{\text {ads }}^{\mathrm{H}_{2} \mathrm{O}-\mathrm{M}, \text { nlc }}$ \\
\hline $\mathrm{Cu}(110)$ & $-724(-642)$ & -291 & -433 & -488 & -128 & -360 \\
$\mathrm{Ru}(0001)$ & $-783(-700)$ & -322 & -462 & -536 & -158 & -378 \\
\hline \hline
\end{tabular}

monolayers the intact water overlayer exhibits a sharp $\sqrt{3}$ diffraction pattern in low-energy electron diffraction (LEED) but a very low specular reflectivity in helium atom scattering (HAS). Although the precise structure of the overlayer has not been resolved, a compelling model that explains both the order observed in LEED and the apparent disorder from HAS has been put forward. The overlayer involves water adsorbed near Ru atop sites in a hydrogen bonded honeycomb network, containing chains of flat-lying and chains of H-down bonded water in a hexagonal superstructure. Here, we have considered this extended chains model in a $2 \sqrt{3} \times \sqrt{3}$ unit cell (Fig. 1(c)), as well as intact H-down (Fig. 1(d)) and H-up $\sqrt{3} \times \sqrt{3}$ bilayers. With PBE the chains are more stable than the bilayers but are less stable than bulk ice (Fig. 2 and Table SII) [34]. Therefore, as with water on $\mathrm{Cu}(110)$, with PBE one would not expect the formation of an extended overlayer. However, when vdW interactions are accounted for the situation is reversed. In particular, optB88-vdW predicts that the extended chain structure is indeed $72 \mathrm{meV} / \mathrm{H}_{2} \mathrm{O}\left(97 \mathrm{meV} / \mathrm{H}_{2} \mathrm{O}\right.$ if zero point energy is included) more stable than bulk ice. These results are in apparent agreement with experiment, where wetting is observed for an intact water overlayer. Again the structures obtained from PBE and optB88-vdW do not differ to any great extent (all distances are within $0.02 \AA)$.

We now discuss why dispersion improves the relative energies of the overlayers and bulk ice. A decomposition of the total adsorption energy in to water-water and water-metal contributions proves useful (see the supporting information for details). With PBE the total adsorption energy is split equally between water-water and water-metal bonding (Fig. 
2). When we switch to optB88-vdW, the adsorption energy increases by about 150 and $180 \mathrm{meV}$ for $\mathrm{Cu}$ and $\mathrm{Ru}$, respectively. Crucially this increase comes almost exclusively from water-metal bonding, with essentially no change in water-water bonding (Fig. 2). Further analysis shows that this increase in water-metal bonding is largely due to non-local correlation. Indeed, on both $\mathrm{Cu}$ and $\mathrm{Ru}$ the non-local contribution to the water-metal bonding exceeds that for the water-water bonding by a factor of 2 to 3 (Table II). It can also be seen from Table \ that the non-local contribution to the water-metal bonding on $\mathrm{Ru}$ is larger than that on $\mathrm{Cu}$, which explains why dispersion has a larger impact on $\mathrm{Ru}$ than on $\mathrm{Cu}$. Although, it is somewhat counterintuitive that dispersion favours the formation of $1 \mathrm{D}$ and $2 \mathrm{D}$ structures over $3 \mathrm{D}$ bulk ice, our analysis is consistent with the fact that $\mathrm{Ru}$ and $\mathrm{Cu}$ atoms have much larger polarizabilities than $\mathrm{O}$ and $\mathrm{H}$. Specifically, the relative $C_{6}$ coefficients from time-dependent DFT calculations [35] of $\mathrm{Ru}: \mathrm{Cu}: \mathrm{O}: \mathrm{H}$ are $79: 44: 2$ : 1 , respectively.

Before concluding, we stress that although progress has been made we are still some way from quantitative first principles predictions of wetting and related phenomena such as heterogeneous ice nucleation rates. In particular, here we have obtained an improved description of the relative energies of water-ice overlayers and bulk ice, but this is achieved at the expense of a slightly worse absolute lattice energy for ice. The experimental lattice energy for bulk ice Ih is $610 \mathrm{meV} / \mathrm{H}_{2} \mathrm{O}$ [16], our $\mathrm{PBE}$ value is $-663 \mathrm{meV} / \mathrm{H}_{2} \mathrm{O}$, and our optB88vdW value is $-711 \mathrm{meV} / \mathrm{H}_{2} \mathrm{O}$. PBE outperforms optB88-vdW on the lattice energy because the missing dispersion is compensated for by the tendency of PBE to slightly overestimate H-bonds between water molecules which are almost linear [36]. optB88-vdW, on the other hand, is known to overestimate the vdW correlation as the number of H-bonds between water molecules increases [25]. Associated with this overbinding is a lattice constant that is too small: the experimental volume is $32.1 \AA^{3} / \mathrm{H}_{2} \mathrm{O}$, the PBE value is $30.4 \AA^{3} / \mathrm{H}_{2} \mathrm{O}$, and the optB88-vdW value is $29.8 \AA^{3} / \mathrm{H}_{2} \mathrm{O}$. The issue of the lattice constant of bulk ice is particularly relevant to heterogeneous ice nucleation, where epitaxial match between the substrate and ice has long been considered a key factor in the nucleating ability of a substrate. It remains to be seen if non-local vdW functionals in their current form are capable of simultaneously addressing all of these issues. Analysis of the underlying errors in the exchange and (nonlocal) correlation components might help to shed light on this [37]. Although, in the longer term the application of the random phase approximation within the adiabatic connection 
fluctuation dissipation theorem or quantum Monte Carlo to ice-like overlayers on metals would be welcomed.

In conclusion, we have considered the role of dispersion in water-metal bonding. Analysis of the relative contributions of water-water and water-metal bonding, to two wetting layers of contemporary importance, highlights the critical role dispersion plays in the wetting of metals by water. Given that DFT plays a central role in interpreting and understanding the most well-defined experimental studies of wetting, it is satisfying that progress with the long-standing wetting problem has been made. Although dispersion is of minor importance for many questions of structure and bonding, we have shown here that the dispersion forces between water and metals are sufficiently large to favor formation of $1 \mathrm{D}$ and $2 \mathrm{D}$ wetting structures over 3D bulk ice. This arises from the much larger polarizability of the metal atoms compared to oxygen and hydrogen, and so it is likely that dispersion will be of importance to water on metals in general and not just the exemplar systems considered here.

JC acknowledges financial support from the Royal Society through a Newton International fellowship and support from Matthias Scheffler. AM is supported by the EURYI scheme (www.esf.org/euryi), the EPSRC, and the European Research Council. We are grateful for computer time to UCL Research Computing and the UK's national high performance computing service HECToR (from which access was partly obtained via the UK's Material Chemistry Consortium, EP/F067496). We also thank Peter Feibelman and Alexandre Tkatchenko for valuable feedback on a draft of this article.

[1] A. Hodgson and S. Haq, Surf. Sci. Rep. 64, 381 (2009).

[2] M. A. Henderson, Surf. Sci. Rep. 46, 1 (2002).

[3] J. Cerda et al., Phys. Rev. Lett. 93, 116101 (2004).

[4] J. Carrasco et al., Nature Mater. 8, 427 (2009).

[5] S. Nie et al., Phys. Rev. Lett. 105, 026102 (2010).

[6] P. J. Feibelman, Science 295, 99 (2002).

[7] P. J. Feibelman, Nature Mater. 8, 372 (2009).

[8] S. Meng et al., Phys. Rev. Lett. 89, 176104 (2002). 
[9] P. J. Feibelman, Phys. Rev. Lett. 91, 059601 (2003).

[10] A. Michaelides et al., Phys. Rev. Lett. 90, 216102 (2003).

[11] A. Michaelides, A. Alavi, and D. A. King, Phys. Rev. B 69, 113404 (2004).

[12] S. Meng, E. G. Wang, and S. Gao, Phys. Rev. B 69, 195404 (2004).

[13] S. Meng et al., Chem. Phys. Lett. 402, 384 (2005).

[14] J. Ren and S. Meng, J. Am. Chem. Soc. 128, 9282 (2006).

[15] S. Haq et al., Phys. Rev. B 73, 115414 (2006).

[16] P. J. Feibelman, Phys. Chem. Chem. Phys. 10, 4688 (2008); and references therein.

[17] S. Schnur and A. Gross, New J. Phys. 11, 125003 (2009).

[18] P. J. Feibelman, Farad. Discuss. 141, 467 (2009).

[19] M. Gallagher et al., Farad. Discuss. 141, 231 (2009).

[20] P. J. Feibelman, Top. Catal. 53, 417 (2010).

[21] P. J. Feibelman, Phys. Today 63, 34 (2010).

[22] P. J. Feibelman, Phys. Rev. B. 72, 113405 (2005).

[23] I. Hamada, K. Lee, and Y. Morikawa, Phys. Rev. B 81, 115452 (2010).

[24] M. Dion et al., Phys. Rev. Lett. 92, 246401 (2004).

[25] J. Klimeš, D. R. Bowler, and A. Michaelides, J. Phys.: Condens. Matter 22, 022201 (2010).

[26] G. Kresse, J. Hafner, Phys. Rev. B 47, 558 (1993).

[27] G. Kresse, J. Furthmüller, Phys. Rev. B 54, 11169 (1996).

[28] G. Román-Pérez and J. M. Soler, Phys. Rev. Lett. 103, 096102 (2009).

[29] J. P. Perdew, K. Burke, M. Ernzerhof, Phys. Rev. Lett. 77, 3865 (1996); 78, 1396 (1997).

[30] D. R. Hamann, Phys. Rev. B 55, R10157 (1997).

[31] G. Kresse and D. Joubert, Phys. Rev. B 59, 1758 (1999).

[32] T. Yamada et al., Phys. Rev. Lett. 96, 036105 (2006).

[33] J. Lee et al., J. Phys. Chem. C 112, 17672 (2008).

[34] In [15] a slightly larger value $\left(-658 \mathrm{meV} / \mathrm{H}_{2} \mathrm{O}\right)$ was reported for the adsorption energy of the extended chain structure (using PW91, ultra-soft pseudopotentials, and a $396 \mathrm{eV}$ cut-off). Here, we find that with our most tightly converged set-up at the PBE level the chains have an adsorption energy of $-605 \mathrm{meV} / \mathrm{H}_{2} \mathrm{O}$, about $50-60 \mathrm{meV} / \mathrm{H}_{2} \mathrm{O}$ less stable than ice with PBE.

[35] S. Grimme et al., J. Chem. Phys. 132,154104 (2010).

[36] B. Santra, A. Michaelides, and M. Scheffler, J. Chem. Phys. 127, 184104 (2007). 
[37] L. Romaner et al., (submitted) 